\title{
313 - INFORMAL SUPPORT FOR PEOPLE WITH ALZHEIMER'S DISEASE AND RELATED DEMENTIA IN RURAL UGANDA: A QUALITATIVE STUDY
}

Pia N. Nankinga, Jr., BBA, PDG

Background: The generation of people getting older has become a public health concern worldwide. People aged 65 and above are the most at risk for Alzheimer's disease which is associated with physical and behavioral changes. This nurtures informal support needs for people living with dementia where their families together with other community members are the core providers of day to day care for them in the rural setting. Despite global concern around this issue, information is still lacking on informal support delivered to these people with dementia.

Objective: Our study aimed at establishing the nature of informal support provided for people with dementia (PWDs) and its perceived usefulness in rural communities in South Western Uganda.

Methods: This was a qualitative study that adopted a descriptive design and conducted among 22 caregivers and 8 opinion leaders in rural communities of Kabale, Mbarara and Ibanda districts in South Western Uganda. The study included dementia caregivers who had been in that role for a period of at least six months and opinion leaders in the community. We excluded trained health workers.

Results: The study highlights important forms of informal support offered to PWDs such as support in activities of daily living, enabling access to medical attention, recovering misplaced items, provision of herbal remedy, informal counseling, and sourcing caregivers from other families to offer presence and support in the hope to impact positively on behavioral outbursts and the frustration of living with dementia.

Conclusion: The study revealed various forms of informal support that are available for PWDs in South Western Uganda and stressed the role of caregivers and the perceived usefulness of the care provided.

Key words: Informal support, dementia and rural communities. 